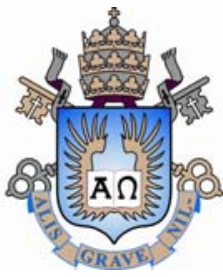

Sandro Rogério Zang

\title{
Aplicação do Método do Casamento de Modos na Análise e no Projeto de Estruturas Coaxiais
}

Dissertação de Mestrado

Dissertação apresentada como requisito parcial para obtenção do título de Mestre pelo Programa de PósGraduação em Engenharia Elétrica da PUC-Rio.

Orientador: José Ricardo Bergmann 


\title{
Pontifícia Universidade Católica \\ DO RIO DE JANEIRO
}

\section{Sandro Rogério Zang}

\section{Aplicação do Método do Casamento de Modos na Análise e no Projeto de Estruturas Coaxiais}

\begin{abstract}
Dissertação apresentada como requisito parcial para obtenção do título de Mestre pelo Programa de PósGraduação em Engenharia Elétrica da PUC-Rio. Aprovada pela Comissão Examinadora abaixo assinada.
\end{abstract}

José Ricardo Bergmann

Orientador

Centro de Estudos em Telecomunicações - PUC-Rio

Fernando José da Silva Moreira Universidade Federal de Minas Gerais - UFMG

Luiz Costa da Silva

Centro de Estudos em Telecomunicações - PUC-Rio

Flávio José Vieira Hasselmann

Centro de Estudos em Telecomunicações - PUC-Rio

José Eugênio Leal

Coordenador(a) Setorial do Centro Técnico Científico - PUC-Rio

Rio de Janeiro, 12 de setembro de 2005. 
Todos os direitos reservados. É proibida a reprodução total ou parcial do trabalho sem autorização da universidade, do autor e do orientador.

\section{Sandro Rogério Zang}

Graduou-se em Engenharia Elétrica, em julho de 2003, na Universidade Federal de Santa Maria. Em agosto do mesmo ano, iniciou no Centro de Estudos em Telecomunicações da Pontifícia Universidade Católica do Rio de Janeiro seu mestrado na área de eletromagnetismo aplicado.

Ficha Catalográfica

Zang, Sandro Rogério

Aplicação do Método do Casamento de Modos na Análise e no Projeto de Estruturas Coaxiais / Sandro Rogério Zang; orientador: José Ricardo Bergmann. Rio de Janeiro: PUC, Departamento de Engenharia Elétrica, 2005.

121 f. il. $30 \mathrm{~cm}$

Dissertação (Mestrado) - Pontifícia Universidade Católica do Rio de Janeiro, Departamento de Engenharia Elétrica.

Inclui referências bibliográficas

1. Engenharia elétrica - Teses. 2. Guias de onda coaxiais. 3. Estruturas de acoplamento. 4. Método do Casamento de Modos. 5. Alimentadores de antenas. 6. Matriz de espalhamento. I. Bergmann, José Ricardo. II. Pontifícia Universidade Católica do Rio de Janeiro. Departamento de Engenharia Elétrica. III. Título. 


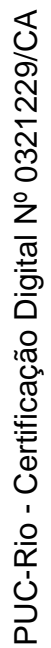

À minha família 


\section{Agradecimentos}

Ao meu orientador José Ricardo Bergmann pelo estímulo, paciência e orientação, fundamentais em todas as etapas do desenvolvimento desse trabalho.

À minha família, em especial aos meus pais pela educação, carinho e incentivo, sem eles nada disso seria possível.

À Monique pelo amor, dedicação e por estar ao meu lado.

Aos amigos Tiago, Julia, Sheila, Andrea e Djeison pelo carinho, companheirismo e amizade sempre presente.

Aos colegas de mestrado, em especial aos amigos do PAA, pelo apoio e colaboração.

À Maria Lúcia e demais funcionários do CETUC, pelo carinho e atenção.

Ao CNPq e à PUC-Rio, pelos auxílios concedidos, sem os quais esse trabalho não poderia ter sido realizado. 


\section{Resumo}

Zang, Sandro Rogério. Aplicação do Método do Casamento de Modos na Análise e no Projeto de Estruturas Coaxiais. Rio de Janeiro, 2005. 121p. Dissertação de Mestrado - Departamento de Engenharia Elétrica, Pontifícia Universidade Católica do Rio de Janeiro.

Neste presente trabalho, o Método do Casamento de Modos é aplicado na análise e no projeto de estruturas coaxiais. A estrutura de excitação é um dos pontos críticos do projeto de antenas do tipo discone quando se pretende explorar suas características de banda larga. Seções não uniformes de guias de ondas coaxiais são usualmente utilizadas como elementos dessa estrutura de excitação sendo, eventualmente, necessária a inclusão de anéis dielétricos para garantir a rigidez mecânica da antena. Devido a essas não uniformidades encontradas nas dimensões e no meio dielétrico desse dispositivo de microondas, métodos numéricos serão utilizados na predição do seu comportamento eletromagnético. O correto dimensionamento desses anéis e das descontinuidades de guias de onda coaxiais que compõe a estrutura de alimentação, permite estabelecer um compromisso entre minimização das perdas, alargamento da banda passante e rigidez mecânica. Associados ao Método do Casamento de Modos serão utilizados algoritmos de otimização que farão o ajuste das dimensões dessas seções de guias de onda coaxiais não uniformes, visando à minimização da perda de retorno e o aumento da banda de operação da antena. Finalmente, será feito o estudo de algumas estruturas de excitação, onde serão otimizados seus desempenhos para a perda de retorno.

\section{Palavras-chave}

Descontinuidades em guias de onda coaxiais; método do Casamento de Modos; matriz de espalhamento; estruturas de acoplamento. 


\section{Abstract}

Zang, Sandro Rogério. Application of Mode Matching Technique in the Analysis and Project of Coaxial Structures. Rio de Janeiro, 2005. 121p. MSc. Dissertation - Departamento de Engenharia Elétrica, Pontifícia Universidade Católica do Rio de Janeiro.

In this present work, the Mode Matching technique is applied in the analysis and project of coaxial structures. The feed junction is one of the critical points of the project of discone antennas when broadband performance is expected. Usually, these junctions are composed of nonuniform sections of coaxial waveguide and, to guarantee the necessary mechanical rigidity of the structure, some sections are filled with dielectric material. The project of these feed junctions requires a compromise between frequency band, compact structure, minimal losses, and mechanical rigidity. Due to the discontinuities, numerical methods are used to predict the electromagnetic behavior. Here, Mode Matching technique associated with an optimization algorithm is employed in the adjustment of the dimensions of the coaxial waveguide sections, seeking the minimization of the return loss and the enlargement of the antenna bandwidth. Several types of junctions are explored and their performance is compared.

\section{Keywords}

Coaxial waveguide discontinuities; Mode Matching technique; scattering matrix; coupler structures. 


\section{Sumário}

1 Introdução

2 Determinação dos Campos Modais para o Guia de Onda Coaxial 20

2.1. Introdução 20

2.2. Solução da equação de Onda Homogênea em Coordenadas Cilíndricas 21

2.2.1. Modo Transversal Magnético $\left(T M^{2}\right) \quad 26$

2.2.2. Modo Transversal Elétrico $\left(T E^{z}\right)$

2.2.3. Modo Transversal Eletromagnético $\left(T E M^{z}\right)$

2.3. Modos $T M^{Z}$ e $T E^{z}$ para $l=0 \quad 37$

3 Método do Casamento de Modos 39

3.1. Introdução 39

3.2. Matriz de Espalhamento de uma Descontinuidade de Seção Transversal de Guia de onda coaxial 41

3.2.1. Cálculo dos Elementos da Matriz $[\mathrm{P}]$, para $l=0$

3.2.2. Cálculo dos Elementos da Matriz $[R]$ para $l=0$

3.2.3. Cálculo dos Elementos da Matriz [Q], para $l=0$

3.3. Cascateamento Progressivo das Matrizes de Espalhamento de Várias Descontinuidades 56

3.3.1. Matriz de Espalhamento de um Trecho de Guia de Onda Coaxial liso 57

3.3.2. Matriz de Espalhamento da Associação em Cascata de duas

Descontinuidades 58

3.4. Validação do Algoritmo Numérico Implementado 63

3.4.1. Primeiro caso 63

$\begin{array}{ll}\text { 3.4.2. Segundo caso } & 65\end{array}$

4 Estudo de Estruturas de Acoplamento 68

4.1. Introdução 68

4.2. Primeira estrutura de acoplamento 69

4.2.1. Otimização da Estrutura de Acoplamento 76

4.2.2. Otimização das Dimensões dos Guias de Onda Coaxiais Lisos que 
$\begin{array}{ll}\text { Compõem a Estrutura de Acoplamento } & 78\end{array}$

4.2.3. Comparação entre Simulações e Medidas para Perda de Retorno 84

4.3. Segunda estrutura de acoplamento 86

4.4. Terceira estrutura de acoplamento 96

4.4.1. Dimensionamento das Estruturas de Suporte Mecânico 97

4.4.2. Dimensionamento da Estrutura de Transição 100

4.4.3. Dimensionamento da Estrutura de Acoplamento Completa 102

4.5. Quarta estrutura de acoplamento 107

4.5.1. Dimensionamento das Estruturas de Suporte Mecânico 108

4.5.2. Dimensionamento da Estrutura de Acoplamento Completa 114

$\begin{array}{ll}5 \text { Conclusão } & 117\end{array}$

6 Referências Bibliográficas 121 


\section{Lista de figuras}

Figura 1.1 - Alimentação de uma antena discone modificada, através de um guia de onda coaxial.

Figura 2.1 - Geometria do problema proposto. (a) seção de guia de onda coaxial e (b) sistema de coordenadas cilíndricas.

21

Figura 2.2 - Representação dos vetores unitários em coordenadas cilíndricas para dois pontos distintos $\left(\rho_{1}, \phi_{1}, z_{1}\right)$ e $\left(\rho_{2}, \phi_{2}, z_{2}\right)$.

Figura $2.3-f_{c_{l m}}^{T M}$ em função de $a / b$ para os primeiros modos $T M_{l m}^{2}$, obtidos através da solução numérica da equação (2.39), considerando $b=15 \mathrm{~mm}$.

Figura 2.4 - Distribuição das componentes transversais de campo no interior do guia de onda coaxial para o modo $T M_{01}^{z}$.

Figura $2.5-f_{c_{l m}}^{T E}$ em função de $a / b$ para os primeiros modos $T E_{l m}^{z}$, obtidos através da solução numérica da equação (2.75), considerando $b=15 \mathrm{~mm}$.

Figura 2.6 - Distribuição das componentes transversais de campo no interior do guia de onda coaxial para os modos (a) $T E_{11}^{z}$ e (b) $T E_{21}^{z}$. 34 Figura 2.7 - Distribuição das componentes transversais de campo no interior do guia de onda coaxial para o modo TEM ${ }^{z}$.

Figura 3.1 - Estrutura de acoplamento entre dois guias de onda coaxiais infinitos de dimensões arbitrárias. (a) Visão espacial e (b) seção longitudinal da estrutura de acoplamento.

40

Figura 3.2 - llustração dos modos refletidos e transmitidos em cada descontinuidade de guia de onda coaxial.

Figura 3.3 - Descontinuidade em guia de onda coaxial: (a) seção longitudinal e (b) seção transversal.

Figura 3.4 - Ilustração das amplitudes dos campos incidentes e espalhados na descontinuidades $(z=0)$. 42

Figura 3.5 - Descontinuidade decrescente em guia de onda coaxial. 49 Figura 3.6 - (a) representação das duas matrizes de espalhamento conectadas, (b) representação da matriz de espalhamento geral obtida pelo cascateamento.

Figura 3.7 - Estrutura de acoplamento entre dois guias de onda coaxiais de dimensões distintas. 
Figura 3.8 - $\left|S 11_{00}\right|(d B)$ para a estrutura de acoplamento da Figura 3.7.

Figura 3.9 - Estrutura de acoplamento entre dois guias de onda coaxiais de dimensões iguais, utilizando carregamento dielétrico.

Figura 3.10 - $\left|S 11_{00}\right|(d B)$ para a estrutura de acoplamento da Figura 3.9. 67

Figura 4.1 - Alimentação de uma antena discone modificada, através de um guia de onda coaxial.

69

Figura 4.2 - Estrutura de acoplamento entre dois guias de onda coaxiais de dimensões diferentes.

Figura 4.3 - Estrutura de acoplamento com deslocamento $G$ no cilindro condutor central (a) para a direita e (b) para a esquerda.

Figura 4.4 - Convergência dos valores de $\left|S 11_{00}\right|(d B)$ em função do número máximo $N$ de modos $T M_{0 n}$, para a freqüência de $1 \mathrm{GHz}$, considerando (a) $G=0.1 \mathrm{~mm}$, (b) $G=10^{-2} \mathrm{~mm}$, (c) $G=10^{-3} \mathrm{~mm}$ e (d) $G=10^{-4} \mathrm{~mm}$, da estrutura de acoplamento ilustrada na Figura 4.3.

Figura 4.5 - Convergência dos valores de $\left|S 11_{00}\right|(d B)$ em função do número máximo $N$ de modos $T M_{0 n}$, para a freqüência de $3 \mathrm{GHz}$, considerando (a) $G=0.1 \mathrm{~mm}$, (b) $G=10^{-2} \mathrm{~mm}$, (c) $G=10^{-3} \mathrm{~mm}$ e (d) $G=10^{-4} \mathrm{~mm}$, da estrutura de acoplamento ilustrada na Figura 4.3.

Figura 4.6 - $\left|S 11_{00}\right|(d B)$ para a estrutura de acoplamento ilustrada na Figura 4.2, considerando o deslocamento para a direita e esquerda e (a) $L=10 \mathrm{~mm}$, (b) $L=12.5 \mathrm{~mm}$ e (c) $L=15 \mathrm{~mm}$.

Figura 4.7 - $\left|S 11_{00}\right|(d B)$ para o acoplador da Figura 4.2, considerando-se $L_{i}=10,12.5$ e $15 \mathrm{~mm}$.

Figura 4.8 - Visualização das perdas parciais referentes a cada descontinuidade.

Figura 4.9 - Visualização da função objetivo $F(x)$. 77

Figura 4.10 - Curvas descritas pela equação (4.7) em função de $\gamma$. 78

Figura 4.11 - Comprimento dos guias de onda coaxiais que compõem o acoplador em função da curvatura descrita pela equação (4.8) aplicada ao cilindro condutor externo do acoplador.

Figura 4.12 - Comparação do $\left|S 11_{00}\right|(d B)$ da estrutura inicial de otimização e da estrutura otimizada com o valor especificado para a perda de retorno, $P^{o b j}=-45 d B$, considerando-se acopladores com (a) $N=2$ (b) $N=3$ e (c) $N=4$ seções de guias de onda intermediárias compondo a estrutura, sendo $L_{T}=20 \mathrm{~mm}$. 
Figura 4.13 - Comparação do $\left|S 11_{00}\right|(d B)$ da estrutura inicial de otimização e da estrutura otimizada com o valor especificado para a perda de retorno, $P^{o b j}=-45 d B$, considerando-se acopladores com (a) $N=2$ (b) $N=3$ e (c) $N=4$ seções de guias de onda intermediárias compondo a estrutura, sendo $L_{T}=30 \mathrm{~mm}$.

Figura 4.14 - Estrutura de acoplamento simétrica. 84

Figura 4.15 - Estrutura de acoplamento simétrica após a inclusão do deslocamento no condutor central.

Figura 4.16 - Comparação entre $\left|S 11_{00}\right|(d B)$, obtido nas medidas, e simulação para o dispositivo da figura 4.14, considerando-se $L_{1}=L_{2}=L_{3}=10 \mathrm{~mm}$.

Figura 4.17 - Comparação entre $\left|S 11_{00}\right|(d B)$, obtido nas medidas, e simulações para o dispositivo da figura 4.15, considerando-se $L_{1}=L_{2}=L_{3}=10 \mathrm{~mm}$ e $G$ variando de $0.1 \mathrm{a} 1 \mathrm{~mm}$.

86

Figura 4.18 - Estrutura de acoplamento entre dois guias de onda coaxiais de dimensões diferentes, com anéis dielétricos (a) depois e (b) antes e depois do acoplador.

Figura 4.19 - $\left|S 11_{00}\right|(d B)$ para a estrutura de acoplamento ilustrada na Figura 4.18(a), para (a) $L d=1 \mathrm{~mm}$, (b) $L d=2 \mathrm{~mm}$ e (c) $L d=3 \mathrm{~mm}$. Figura 4.20 - Comparação do $\left|S 11_{00}\right|(d B)$ da estrutura inicial de otimização e da estrutura otimizada com o valor especificado para a perda de retorno, $P^{o b j}=-45 d B$, considerando-se (a) $L d=1 \mathrm{~mm}$, (b) $L d=2 \mathrm{~mm}$ e (c) $L d=3 \mathrm{~mm}$ para a estrutura de acoplamento ilustrada na Figura 4.18(a). Figura 4.21 - Comparação do $\left|S 11_{00}\right|(d B)$ da estrutura inicial de otimização e da estrutura otimizada, referentes a segunda etapa de otimização, com o valor especificado para a perda de retorno, $P^{o b j}=-45 d B$, considerando-se (a) $L d=1 \mathrm{~mm}$, (b) $L d=2 \mathrm{~mm}$ e (c) $L d=3 \mathrm{~mm}$ para a estrutura de acoplamento ilustrada na Figura 4.18(a).

Figura 4.22 - $\left|S 11_{00}\right|(d B)$ para a estrutura de acoplamento ilustrada na Figura 4.18(b), para (a) $L d=1 \mathrm{~mm}$, (b) $L d=2 \mathrm{~mm}$ e (c) $L d=3 \mathrm{~mm}$.

Figura 4.23 - Comparação do $\left|S 11_{00}\right|(d B)$ da estrutura inicial de otimização e da estrutura otimizada, com o valor especificado para a perda de retorno, $P^{o b j}=-45 d B$, considerando-se (a) $L d=1 \mathrm{~mm}$, (b) $L d=2 \mathrm{~mm}$ e (c) $L d=3 \mathrm{~mm}$ para a estrutura de acoplamento ilustrada na Figura 4.18(b). 
Figura 4.24 - Comparação do $\left|S 11_{00}\right|(d B)$ da estrutura inicial de otimização e da estrutura otimizada, referentes a segunda etapa de otimização, com o valor especificado para a perda de retorno, $P^{o b j}=-45 d B$, considerando-se (a) $L d=1 \mathrm{~mm}$, (b) $L d=2 \mathrm{~mm}$ e (c) $L d=3 \mathrm{~mm}$ para a estrutura de acoplamento ilustrada na Figura 4.18(b).

Figura 4.25 - Estrutura de acoplamento baseada na combinação das estruturas dos itens 3.4.1 e 3.4.2.

Figura 4.26 - Estrutura de suporte.

Figura 4.27 - $\left|S 11_{00}\right|(d B)$ (a) para o primeiro suporte e (b) para o segundo suporte ilustrados na Figura 4.26.

Figura 4.28 - Comparação entre $\left|S 11_{00}\right|(d B)$ da estrutura inicial de otimização e da estrutura otimizada e o valor especificado para a perda de retorno, $P^{o b j}=-50 d B$, (a) para o primeiro suporte e (b) para o segundo suporte, ilustrados na Figura 4.26.

100

Figura 4.29 - Estrutura de transição entre dois guias de onda coaxiais de dimensões distintas.

100

Figura 4.30 - $\left|S 11_{00}\right|(d B)$ para a estrutura de transição ilustrada na Figura 4.29.

101

Figura 4.31 - Comparação entre $\left|S 1_{00}\right|(d B)$ da estrutura inicial de otimização e da estrutura otimizada e o valor especificado para a perda de retorno, $P^{o b j}=-50 d B$, referentes à estrutura de transição ilustrada na Figura 4.29. 102 Figura $4.32-\left|S 11_{00}\right|(d B)$ para o acoplador ilustrado na Figura 4.25. 103 Figura 4.33 - Comparação entre $\left|S 11_{00}\right|(d B)$ da estrutura inicial de otimização e da estrutura otimizada e o valor especificado para a perda de retorno, $P^{o b j}=-50 d B$, referentes à estrutura de acoplamento ilustrada na Figura 4.25.104 Figura 4.34 - Comparação entre $\left|S 1_{00}\right|(d B)$ da estrutura inicial de otimização e da estrutura otimizada, referente à segunda de otimização, e o valor especificado para a perda de retorno, $P^{o b j}=-50 d B$, da estrutura de acoplamento ilustrada na Figura 4.25. 105 Figura 4.35 - Comparação entre $\left|S 11_{00}\right|(d B)$ da estrutura inicial de otimização e da estrutura otimizada, referente a terceira etapa de otimização, e o valor especificado para a perda de retorno, $P^{o b j}=-45 d B$, da estrutura de acoplamento ilustrada na Figura 4.25. 107 Figura 4.36 - Estrutura de acoplamento remodelada baseada no item 4.4. 108 
Figura 4.37 - Estrutura de suporte remodelada.

Figura 4.38 - $\left|S 11_{00}\right|(d B)$ para o primeiro suporte, ilustrado na Figura 4.37, para (a) $L_{N}=1 \mathrm{~mm}$, (b) $L_{N}=2 \mathrm{~mm}$ e (c) $L_{N}=3 \mathrm{~mm}$.

109

Figura 4.39 - Comparação entre $\left|S 1_{00}\right|(d B)$ da estrutura inicial de otimização e da estrutura otimizada e o valor especificado para a perda de retorno, $P^{o b j}=-50 d B$, referentes ao primeiro suporte, ilustrado na Figura 4.37, para (a) o primeiro e (b) segundo caso otimizado.

Figura $4.40-\left|S 11_{00}\right|(d B)$ para o segundo suporte, ilustrado na Figura 4.37, para (a) $L_{N}=1 \mathrm{~mm}$, (b) $L_{N}=2 \mathrm{~mm}$ e (c) $L_{N}=3 \mathrm{~mm}$.

112

Figura 4.41 - Comparação entre $\left|S 11_{00}\right|(d B)$ da estrutura inicial de otimização e da estrutura otimizada e o valor especificado para a perda de retorno, $P^{o b j}=-50 d B, \quad$ referentes ao segundo suporte, ilustrado na Figura 4.37, considerando-se $L_{2}=3 \mathrm{~mm}$. 113

Figura 4.42 - $\left|S 11_{00}\right|(d B)$ para o acoplador ilustrado na Figura 4.36. 114

Figura 4.43 - Comparação entre $\left|S 11_{00}\right|(d B)$ da estrutura inicial de otimização e da estrutura otimizada e o valor especificado para a perda de retorno, $P^{o b j}=-50 d B$, referentes à Figura 4.36 .

Figura 4.44 - Comparação entre $\left|S 11_{00}\right|(d B)$ da estrutura inicial de otimização e da estrutura resultante do segundo caso otimizado e o valor especificado para a perda de retorno, $P^{o b j}=-50 d B$, referentes à Figura 4.36. 


\section{Lista de tabelas}

Tabela 3.1 - Dimensões da estrutura de acoplamento da figura 3.7. 64

Tabela 3.2 - Dimensões da estrutura de acoplamento da figura 3.9. 66

Tabela 4.1 - Dimensões dos cilindros condutores interno e externo para o acoplador da Figura 4.2. 70

Tabela 4.2 - Dimensões da estrutura inicial e otimizada para o acoplador com duas seções de guias de onda intermediárias compondo o acoplador, $N=2 \mathrm{e}$ $L_{T}=20 \mathrm{~mm}$.

Tabela 4.3 - Dimensões da estrutura inicial e otimizada para o acoplador com três seções de guias de onda intermediárias compondo o acoplador, $N=3$ e $L_{T}=20 \mathrm{~mm}$.

Tabela 4.4 - Dimensões da estrutura inicial e otimizada para o acoplador com quatro seções de guias de onda intermediárias compondo o acoplador, $N=4 \mathrm{e}$

$L_{T}=20 \mathrm{~mm}$.

Tabela 4.5 - Dimensões da estrutura inicial e otimizada para o acoplador com duas seções de guias de onda intermediárias compondo o acoplador, $N=2 \mathrm{e}$ $L_{T}=30 \mathrm{~mm}$.

Tabela 4.6 - Dimensões da estrutura inicial e otimizada para o acoplador com três seções de guias de onda intermediárias compondo o acoplador, $N=3 \mathrm{e}$ $L_{T}=30 \mathrm{~mm}$.

Tabela 4.7 - Dimensões da estrutura inicial e otimizada para o acoplador com quatro seções de guias de onda intermediárias compondo o acoplador, $N=4$ e $L_{T}=30 \mathrm{~mm}$.

Tabela 4.8 - Dimensões das seções de guias de onda coaxiais referentes à estrutura de acoplamento ilustrada na Figura 4.18.(a) e (b).

Tabela 4.9 - Dimensões das seções de guias de onda coaxiais resultantes da segunda etapa de otimização, para $L d=1 \mathrm{~mm}$, referentes à estrutura de acoplamento ilustrada na Figura 4.18.(a).

Tabela 4.10 - Dimensões das seções de guias de onda coaxiais resultantes da segunda etapa de otimização, para $L d=2 \mathrm{~mm}$, referentes à estrutura de acoplamento ilustrada na Figura 4.18.(a).

Tabela 4.11 - Dimensões das seções de guias de onda coaxiais resultantes da segunda etapa de otimização, para $L d=3 \mathrm{~mm}$, referentes à estrutura de 
acoplamento ilustrada na Figura 4.18.(a).

Tabela 4.12 - Dimensões das seções de guias de onda coaxiais resultantes da segunda etapa de otimização, para $L d=1 \mathrm{~mm}$, referentes à estrutura de acoplamento ilustrada na Figura 4.18.(b).

Tabela 4.13 - Dimensões das seções de guias de onda coaxiais resultantes da segunda etapa de otimização, para $L d=2 \mathrm{~mm}$, referentes à estrutura de acoplamento ilustrada na Figura 4.18.(b).

Tabela 4.14 - Dimensões das seções de guias de onda coaxiais resultantes da segunda etapa de otimização, para $L d=3 \mathrm{~mm}$, referentes à estrutura de acoplamento ilustrada na Figura 4.18.(b).

Tabela 4.15 - Dimensões envolvidas nas estruturas de suporte do acoplador referentes à Figura 4.26.

Tabela 4.16 - Dimensões resultantes dos três casos otimizados, $L_{2}=1,2 e$ $3 m m$, para a primeira estrutura de suporte mecânico.

Tabela 4.17 - Dimensões resultantes dos três casos otimizados, $L_{2}=1,2 \mathrm{e}$ $3 m m$, para a segunda estrutura de suporte mecânico.

Tabela 4.18 - Dimensões da estrutura inicial de otimização para o acoplador completo, ilustrado na Figura 4.25.

Tabela 4.19 - Dimensões resultantes do segundo caso de otimização da estrutura de acoplamento completa referente à Figura 4.25.

Tabela 4.20 - dimensões resultantes do terceiro caso de otimização da estrutura de acoplamento completa referente à Figura 4.25.

Tabela 4.21 - Dimensões resultantes dos dois casos otimizados, $L_{2}=2$ e $3 \mathrm{~mm}$, para a primeira estrutura de suporte mecânico.

Tabela 4.22 - Dimensões resultantes da otimização considerando $L_{2}=3 \mathrm{~mm}$, para a segunda estrutura de suporte mecânico.

Tabela 4.23 - dimensões resultantes do segundo caso de otimização da estrutura de acoplamento completa referente à Figura 4.36. 